NASA

Technical Memorandum 88928
USAAVSCOM

Technical Report 86-C-36

\title{
A Method for Calculating Turbulent Boundary Layers and Losses in the Flow Channels of Turbomachines
}

Lawrence F. Schumann

Propulsion Directorate

U.S. Army Aviation Research and Technology Activity-AVSCOM

Lewis Research Center

Cleveland, Ohio

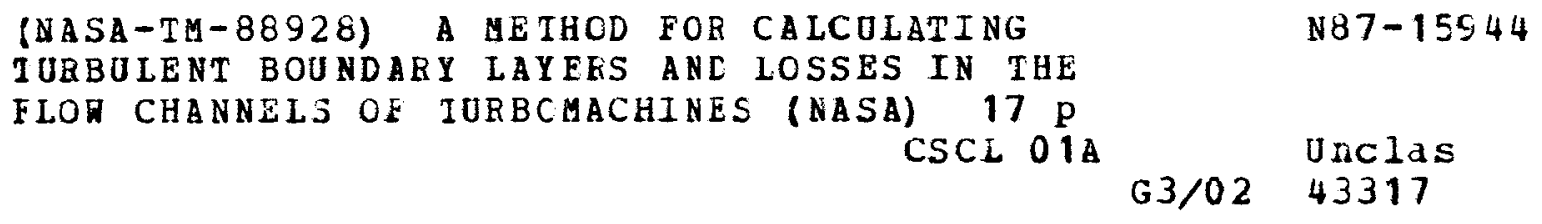

Prepared for the

32nd International Gas Turbine Conference and Exhibition sponsored by the American Society of Mechanical Engineers Anaheim, California, May 31-June 4, 1987 


\author{
Lawrence F. Schumann \\ Propulsion Directorate \\ U.S. Army Aviation Research and Technology Acitivity - AVSCOM \\ Lewis Research Center \\ Cleveland, Ohio 44135
}

\section{ABSTRACT}

An interactive inviscid core flow-boundary layer method is presented for the calculation of turbomachine channel flows. For this method, a one-dimensional inviscid core flow is assumed. The end-wall and blade surface boundary layers are calculated using an inteif gral entrainment method. The boundary layers are

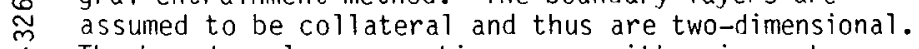
is The boundary layer equations are written in a streamline coordinate system. The streamwise velocity profiles are approximated by power law profiles. Compressibility is accounted for in the streamuise direction but not in the normal direction. Equations are derived for the special cases of conical and two-dimensional rectangular diffusers. For these cases, the assumptions of a one-dimensional core flow and collateral boundary layers are valid. Results using the method are compared with experiment and good quantitative agreement is obtained.

\section{NOMENCLATURE}

$\begin{array}{ll}A_{b} & \text { unblocked flow area }=(1-B) A_{q} \\ A_{g} & \text { geometric area } \\ A_{1}-A_{3} & \text { terms in velocity gradient equation } \\ a & \text { speed of sound } \\ B & \text { blockage } \\ b & \text { passage height } \\ C_{f} & \text { skin friction coefficient } \\ C_{p} & \text { specific heat at constant pressure } \\ C_{p r} & \text { pressure recovery coefficient, } \frac{P-P_{1}}{P_{1 e}-P_{1}}\end{array}$

D diffuser throat diameter

$D_{1}-D_{2}$ terms in velocity gradient equation

$F \quad$ entrainment function

$H_{1} \quad$ shape factor, $\frac{\delta-\delta_{1}}{\delta_{2}}$

$H_{2}$ shape factor, $\frac{\delta_{1}}{\delta_{2}}$

$k_{1}-k_{3}$ terms in area equation

$K_{t}, K_{y}$ curvature terms

L. diffuser length

M relative Mach number, W/a

$M_{u} \quad$ wheel speed Mach number, U/a

$m$ exponent in velocity profile

$m \quad$ mass flux

P pressure

PSTD standard pressure, $101325 \mathrm{~N} / \mathrm{m}$

Re Reynold's number

$\mathrm{R}_{\mathrm{g}}$ gas constant

$r$ radius

s entropy

$T$ temperature

TSTO standard temperature, $288.2 \mathrm{~K}$ 
whee 1 speed

relative velocity

streamline coordinate

normal coordinate

blade number

angle between meridional streamline and axis of rotation

angle between relative velocity vector and meridional plane

specific heat ratio

boundary layer thickness

displacement thickness, $\int_{0}^{\delta}\left(1-\frac{W}{W_{e}}\right) d y$

$\delta_{2}$ momentum thickness, $\int_{0}^{\delta} \frac{W}{W_{e}}\left(1-\frac{W}{W_{e}}\right) d y$

$\theta$ channel divergence angle

$\lambda$ prewhir

$\mu \quad$ coefficient of viscosity

$\rho$ density

T shear stress

$\phi \quad$ entropy function, $\int c_{p} \frac{d T}{T}$

rotational speed

Subscripts:

A spanwise direction

e edge of boundary layer

$r$ radial direction

$t, T$ tangential direction

w wall

$x \quad$ streamline direction

y normal direction

1 channel inlet

2 channel exit

Superscripts

I absolute total conditions

" relative total conditions

\section{INTRODUCTION}

In the preliminary design of turbomachinery it is necessary to estimate the losses in the individual components. For rotors, stators and impellers, this is usually done by using empirical correlations for channel losses (e.g., skin friction, diffusion, etc. (1-4)). For diffusers (conical, rectangular) this is done by empirically relating the diffuser pressure recovery coefficient to the diffuser geometry and inlet flow conditions $(5,6)$. A more rigorous way of estimating these losses would be to calculate the surface boundary layers and from these obtain a massaveraged estimate of channe 1 losses. However, many of the present methods for calculating boundary layers (e.g., the code, BLAYER, ref. 7) are not ideally suited for calculating the boundary layers in turbomachine flow channels.

First of al1, most boundary layer codes are written for external flows, thus, they require a specification of the free-stream velocity distribution. For internal flows, the velocity distribution is not known apriori and thus must be determined by an iterative procedure along with the continuity equation for the assumed inviscid core flow. This can be a very time consuming procedure especially for preliminary design calculations where several levels of iteration must be performed.

Also, most boundary layer codes are written for two-dimensional flows along flat surfaces and thus ignore any coordinate curvature terms (i.e., metrics). This can lead to large errors for some internal channel flows in which the channel end walls are converging or diverging; e.g., nozzles and diffusers.

There have been several methods proposed to calculate the performance of straight-walled, rectangular type diffusers (8-11). These methods are all twodimensional in nature (i.e., they ignore end-wa?! effects) and are limited to the computation of incompressible, straight center-line diffuser flows. These methods are highly developed and predict the performance of this class of diffusers very well even into the stalled regions of the diffuser maps. However, since all these methods are iterative, viscousinviscid interaction methods, their running times can be much longer than that of the method proposed here. This could make them unsuitable for use in compressor preliminary design codes.

For these reasons, a noninterative integralentrainment method for calculating the flow fields in turbomachines has been developed. The method simultaneously calculates the free-stream velocity distribution from an assumed one-dimensional core flow continuity equation together with the equations describing the surface boundary layers. Thus, only one pass through the flow channel is required thereby making the method very fast. The primary assumptions made for the boundary layer calculations are that the flow is basically two-dimensional and that the boundary layer profile can be described by a power law. The exponent in the power 1 aw is allowed to vary as prescribed by the equations of motion. Compressibility is accounted for in the streamline direction but not in the direction normal to the flow surfaces. Turbulent flow is assumed everywhere using the semiempirical Ludwieg-Tillman relation for skin friction. Approximate methods for calculating through regions of fully-developed or separated flows are also presented. Comparisons are made with experimental data for the cases of conical and rectangular diffusers and good agreement is demonstrated for cases where no separation is indicated. Fair agreement is demonstrated in separated regions of the flow. 
The method of analysis used to calculate the flow surface boundary layers in the channel is an integralentrainment method. The method is similar to that used for the calculation of end-wall boundary layers in Ref. 12. For this analysis, the core flow is assumed to be one-dimensional and is described by the one-dimensional continuity and momentum equations.

Two-Dimensional Boundary Layer Method

The following assumptions are made for this analys is:

(1) The flow is steady and turbulent everywhere

(2) The fluid is a perfect gas

(3) The flow in the bourdary layers is twodimensional, i.e., there is no crossflow component of velocity. Therefore, the boundary layers are also collateral, i.e., the flow angle, $B$, is constant through the boundary layer.

(4) The channel flow surfaces are adiabatic

(5) There is no density variation in the direction normal to the channel boundaries al though the density is allowed to vary in the streamline direction, i.e., $\rho=\rho(x)$. This approximation seems to work well for Mach numbers up to 1.0 and slightly beyond (12). For high supersonic flows, some other formulation would have to be used.

(6) The boundary layer velocity profile in the streamline direction may be described by the power-law equation

$$
\frac{w_{x}}{w_{e}}=\left(\frac{y}{\delta}\right)^{m}
$$

where $W_{e}, \delta$, and $m$ are functions of distance along a streamline, $x$, only. The power law approximation adequately predicts pressure recovery up to separation, although the actual profile deviates from the power law approximation more significantly as separation is approached.

To completely solve the problem, the boundary layer thickness, $\delta$, and the boundary layer exponent, $m$, for each surface boundary layer calculated; the free-stream velocity, $W_{e}$; and the free-stream pressure, density and temperature must be determined. Thus, a total of $(4+2 n)$ equations must be solved where $n$ is the number of surface boundary 1 ayers.

If a streamline coordinate system is chosen (with $x$ in the streamline direction, $y$ in the normal to the wall direction, and $t$ in the crossflow direction completing the orthogonal triad), the boundary layer equations for motion can be written as follows:

\section{Continuity}

$$
\frac{\partial\left(\rho W_{x}\right)}{\partial x}+\frac{\partial\left(\rho W_{y}\right)}{\partial y}+\rho W_{x}\left(K_{y}+K_{t}\right)=0
$$

Momentum

$\rho W_{x} \frac{\partial W_{x}}{\partial x}+\rho W_{y} \frac{\partial W_{x}}{\partial y}-\rho \omega_{\omega}^{2} \sin \alpha \cos \beta=-\frac{\partial p}{\partial x}+\frac{1}{r} \frac{\partial\left(r_{x}\right)}{\partial y}$

$$
\frac{\partial p}{\partial y}=0
$$

In Eq. (2), the curvature term, $K_{y}$, is defined as the curvature of the normal coordinate, $y$, in the $t=$ constant $p l$ ane and the curvature term, $k_{t}$, is defined as the curvature of the tangential coordinate, $t$, in the $y=$ constant plane. The normal coordinate is assumed to be everywhere normal to the flow.

If Eqs. (2) and (3) are integrated through the boundary layer with the additional assumption that $K_{t}$, $K_{y}, \alpha, B$ and $r$ are independent of the norma? coordinate, $y$; the following equations result:

$$
\int_{0}^{\delta} \frac{\partial\left(\rho W_{x}\right)}{\partial x} d y+\int_{0}^{\delta} \frac{\partial\left(\rho W_{y}\right)}{\partial y} d y+\left(K_{y}+K_{t}\right) \int_{0}^{\delta} \rho W_{x} d y=0
$$

$$
\begin{array}{r}
\int_{0}^{\delta} \frac{\partial\left(\rho W_{x}^{2}\right)}{\partial x} d y+\rho W_{e} W_{y e}+\rho\left(K_{y}+K_{t}\right) \int_{0}^{\delta} W_{x}^{2} d y \\
-\rho r \delta \omega^{2} \sin \alpha \cos \beta=-\delta \frac{\partial p}{\partial x}-{ }^{\tau} x \omega
\end{array}
$$

Equation (3) can also be evaluated for the isentropic core flow which results in the following equation

$$
\frac{d p}{d x}=-\rho w_{e} \frac{d W_{e}}{d x}+\rho r \omega^{2} \sin \alpha \cos B
$$

One additional unknown was added in the integration of Eq. (3), i.e., the normal velocity at the edqe of the boundary layer, Wye, as shown in Eq. (6). This can be modelled using Head's entrainment relation as was demonstrated in Ref. 12 . The resulting relation is given by

$$
F \equiv \frac{1}{w_{e}}\left(w_{e} \frac{d \delta}{d x}-w_{y e}\right)=0.0306\left(H_{1}-3\right)^{-0.653}
$$

Using the velocity profile from Eq. (1) and performing the indicated differentiations and integrations, Eqs. (5) to (8) can be combined to yield the following equations

$\frac{d m}{d x}=-2 m(1+m)(1+2 m)\left[\frac{1}{W_{e}} \frac{d W_{e}}{d x}+\frac{F}{2 \delta}-\frac{1+2 m}{4 m \delta} c_{f}\right]$

$\frac{1}{\delta} \frac{d \delta}{d x}=-\frac{\left(1-M_{e}^{2}\right)}{w_{e}} \frac{d w_{e}}{d x}+\frac{1}{(1+m)} \frac{d m}{d x}+(1+m) \frac{F}{\delta}$

$$
-\frac{\sin \alpha \cos \beta}{r} M_{u}^{2}-\left(k_{y}+k_{t}\right)
$$

Equations (9) and (10) can be solved if the variation in free stream velocity, $W_{e}$, is known. A detailed derivation of equations similar to Eqs. (9) and (10) is shown in the Appendix to Ref. 12 .

In order to solve these equations, the skin friction coefficient, $C_{f}$, and the entrainment function, $F$, must be evaluated. The skin friction coefficient is obtained from the Ludwieg-Tillman relation which is 


$$
C_{f}=0.246 \operatorname{Re}_{\delta_{2}}-0.268 \mathrm{e}^{-1.561 \mathrm{H}_{2}}
$$

where $H_{2}=1+2 m$.

The entrainment function is a function only of a boundary layer shape factor and was shown in Ref. 12 to be given by

$$
F=0.0306\left(\frac{m}{1-m}\right)^{0.653}
$$

The variation in the free stream velocity, $W_{e}$, can be determined if a one-dimensional core flow is assumed. In this case, the velocity can be determined from the continuity equation for the core flow which can be written

$$
\text { in }=\rho W_{e} A_{b}
$$

where $A_{b}$ represents the unblocked channel area normal to the flow.

Using the results from Eq. (7) and differentiating Eq. (13) results in the equation

$$
\frac{1}{W_{e}} \frac{d W_{e}}{d x}=\frac{1}{\left(1-M_{e}^{2}\right)}\left(-\frac{M_{u}^{2} \sin \alpha \cos \beta}{r}-\frac{1}{A_{b}} \frac{d A_{b}}{d x}\right)
$$

The static temperature of the core flow can be determined from the energy equation for the core flow which is

$$
T=T^{\prime \prime}-\frac{W^{2}}{2 C_{p}}
$$

where

$$
T^{\prime \prime}=T_{1}-\frac{2 \omega \lambda-(\omega r)^{2}}{2 c p}
$$

The static pressure and density can then be determined from either Eq. (7) and the equation of state for the core flow, or from the isentropic relations for the core flow. These equations together with Eq. (14), and Eqs. (9) and (10) written for each boundary layer, then provide the $(4+2 n)$ equations needed to solve the channel flow field.

\section{Fully Developed and Separated Flow}

When the boundary layers from opposite walls of a channel merge, an isentropic core flow no longer exists and the flow is said to be fully developed. In this case, the thin shear layer assumption used in the derivation of Eqs. (2) to (4) is no longer valid. However, it would be useful to carry out the calculations after the flow becomes fully developed. In order to do this, some simplifying assumptions must be made. These are as follows:

(1) The boundary layer Eqs. (2) to (4) describe approximately the fully deveioped flow field.

(2) The centerline of the flow remains isentropic. Although this is consistent with Prandt)'s mixing length theory of turbulence; in reality, it is not valid due to a nonzero shear stress gradient at the centerline.

With these assumptions and the fact that the entrainment function equals zero (as can be seen from the definition in Eq. (8)), and the boundary layer thickness equals half the channel height, Eqs. (9) and (14) can be solved as before.

It would also be useful to be able to continue the calculation beyond an indicated point of boundary layer separation. This point is usually determined by a limiting value of the shape factor, $H_{2}=1+2 \mathrm{~m}$. once again, the boundary layer Eqs. (2) to (4) become invalid when the boundary layer separates. Also, the assumed power law profiles cannot accurately describe a separated flow. However, calculations show that the pressure recovery and losses can be reasonably predicted using some simplifyina assumptions. These are as follows:

(1) The boundary layer Eqs. (2) to (4) and oower law profile remain valid for separated flow.

(2) The skin friction coefficient qoes to zero.

(3) The entrainment function reaches a limiting value chosen to be the value at the separation point.

(4) The shape factor is allowed to increase beyond the separation point.

With these assumptions, the calculations ran proceed beyond points of separation or fully developed flow.

\section{Loss Calculations}

Since the flow is assumed to be two-dimensional, the losses can be determined by a simple mass averaqing procedure. Since the channel exit static pressure is determined in the solution of the boundary layer flow, all that is needed is a static enthalpy corresponding to a mass-averaged flow in the channel in order to calculate the mass-averaged entropy rise from the equation

$$
s_{2}-s_{1}=\phi_{2}-\phi_{1}-R_{q} \ln \frac{P_{2}}{P_{1}}
$$

\section{APPLICATIONS OF THE METHOD}

The method has been applied to the cases of conical and rectangular diffusers. In these cases, the assumptions of a one-dimensional core flow and collateral boundary layers are valid at least for nonseparated flows.

\section{Conical Diffuser}

The basic geometry of a conical diffuser is shown in Fig. 1. In order to solve Eqs. (9), (10) and (14), the cross-sectional area, $A_{b}$, and the curvatures, $K_{y}$ and $K_{t}$, must be determined. The flow area is assumed to ije on a spherical segment as shown which is approximately everywhere normal to the flow. The unblocked flow area is then given by

$$
A_{b}=2 \pi x^{i}\left(1-\cos \left[\theta-\frac{\delta_{l}}{y}\right)\right]
$$

and the area term in Eq. (14) becomes

$$
\frac{1}{A_{b}} \frac{d A_{b}}{d x}=K_{2}-K_{1} \frac{d \delta_{1}}{d x}
$$

where $k_{1}=\frac{\sin \left(\theta-\frac{\delta_{1}}{x}\right)}{x\left(1-\cos \left(\theta-\frac{\delta_{1}}{x}\right)\right)}$ 


$$
K_{2}=\frac{2}{x}+K_{1} \frac{\delta_{1}}{x}
$$

The curvature terms for this case are qiven by

$$
K_{y}=K_{t}=\frac{1}{x}=\frac{\sin \theta}{r}
$$

Then for this case, since $M_{u}=0$, and

$$
\frac{d \delta}{d x}=\frac{m}{1+m} \frac{d \delta}{d x}+\frac{\delta}{(1+m)^{2}} \frac{d m}{d x}
$$

Eq. (14) becomes

$$
\frac{1}{W_{e}} \frac{d W_{e}}{d x}=\frac{A_{1}}{D_{1}}
$$

where $A_{1}=-\frac{\left(2+3 k_{1} \delta_{1}\right)}{x}-2 k_{1} m^{2} F+k_{1}(1+2 m)^{2} \frac{{ }^{c} f}{2}$

$$
D_{1}=\left(1-M_{e}^{2}\right)\left(1+K_{1} \delta_{1}\right)+2 K_{1} \delta_{1}(1+m)(1+2 m)
$$

Equations (9) and (10) complete the system of 3 equations in 3 unknowns $\left(W_{e}, m, \delta\right)$. The equations are solved using a fourth-order Runge-Kutta technique (13). Results using this method have been compared with experimental data from a wind-tunnel diffuser section (14) and with data for conical diffusers (5).

Figures 2 to 4 show comparisons of velocity profiles, static pressure coefficients, and displacement thicknesses at the exit of the cross-lea diffuser of Ref. 14 for three different inlet Reynold's numbers and Mach numbers. In all cases the agreement is good. For these cases, the boundary layers remained attached according to the analysis. Figures $5(a)$ to (d) shows comparisons of pressure recovery coefficient for several arbitrary cases of inlet Mach number, blockage, and cone angle for the conical diffuser data of Ref. 5. Almost all of the data 7 ie in a region where the flow would be expected to be separated according to the analysis. However, the agreement between experiment and analysis is still fair.

\section{Rectangular Diffuser}

The geometry for a rectangular diffuser is shown in Fig. 6. For this case, the boundary layers in the tangential (blade to blade) and spanwise (hub to shroud) directions cannot be assumed to be equal due to the fact that the initial boundary layer thicknesses may be unequal and the flow may become fully developed in one direction only. Thus, the tangential and spanwise boundary layers must be calculated separately. However, the core flow is still assumed to be one-dimensional.

The unblocked flow area for this case is given by

$$
A_{b}=\left(b-2 \delta_{1 A}\right)\left(2 \theta x-2 \delta_{1 T}\right)
$$

and the term in Eq. (12) becomes

$$
\frac{1}{A_{b}} \frac{d A_{b}}{d x}=K_{3}-K_{2} \frac{d \delta_{1 T}}{d x}-K_{1} \frac{d \delta_{1 A}}{d x}
$$

where

$$
\begin{aligned}
& K_{1}=\frac{2}{\left(b-2 \delta_{1 A}\right)} \\
& K_{2}=\frac{1}{\left(\theta x-\delta_{1 T}\right)} \\
& K_{3}=\frac{\theta}{\left(\theta x-\delta_{1 T}\right)}
\end{aligned}
$$

The curvature terms for the tangential boundary layers are given by

$$
k_{y}=\frac{1}{x} ; \quad k_{t}=0
$$

The curvature terms for the spanwise boundary layers are given by

$$
k_{y}=0 ; \quad k_{t}=\frac{1}{x}
$$

Equation (14) can then be expressed

$$
\frac{1}{W_{e}} \frac{d W_{e}}{d x}=\frac{A_{1}+A_{2}+A_{3}}{D_{1}+D_{2}}
$$

where

$$
\begin{aligned}
& A_{1}=-2 K_{2} m_{T}^{2} F_{T}-2 K_{1} m_{A}^{2} F_{A} \\
& A_{2}=\frac{K_{2}}{2}\left(1+2 m_{T}\right)^{2} c_{f T}+\frac{K_{1}}{2}\left(1+2 m_{A}^{2}\right) c_{f A} \\
& A_{3}=-K_{3}-K_{2} \frac{\delta_{1 T}}{x}-K_{1} \frac{\delta_{1 A}}{x} \\
& D_{1}=\left(1-M_{e}^{2}\right)\left(1+K_{2} \delta_{1 T}+K_{1} \delta_{1 A}\right) \\
& D_{2}=2 K_{2} \delta_{1 T}\left(1+m_{T}\right)\left(1+2 m_{T}\right)+2 K_{1} \delta_{1 A}\left(1+m_{A}\right)\left(1+2 m_{A}\right)
\end{aligned}
$$

Equations (9) and (10) written for both the tangential and spanwise boundary layers then complete the system of 5 equations in 5 unknowns $\left(W_{e}, m_{A}, \delta_{A}, m_{T}, \delta T\right)$. Results using this method have been compared with experimental data for rectangular diffusers (6). A comparison of calculated and experimental pressure coefficients for several arbitrary cases of inlet Mach number, blockage, and divergence angle, $\theta$, is shown in Fig. 7(a) to (d). The cases shown correspond to an inlet aspect ratio of one; however, the method is applicable for any arbitrary inlet aspect ratio. The cases were calculated assuming equal boundary layer thicknesses and exponents on the inlet vane surfaces and end walls. The results show fair agreement between calculation and experiment even though the calculations indicate that almost all of the data shown lie in a region where the flow would be separated.

\section{CONCLUSIONS}

An analytical method has been developed to calculate boundary layers and losses in turbomachine flow channels. Results using this method have been compared with experimental data for the special cases of conical and rectangular diffusers. Good arreement is demonstrated for the flow regimes where the analysis indicates no separation. Fair agreement is 
demonstrated for the pressure coefficients calculated in the separated regions of the flow.

Since the method is a noninterative integral method, it is very fast compared to other methods of analysis. Thus, it can be easily adapted for use in preliminary design or off-design codes for turbomachinery components.

\section{REFERENCES}

1. Galvas, M.R., "Analytical Correlation of Centrifugal Compressor Design Geometry for Maximum Efficiency with Specific Speed," NASA TN D-6729, 1972.

2. Crouse, J.E., and Gorrel1, W.T., "Computer Program for Aerodynamic and Blading Design of Multistage Axial-Flow Compressors," NASA TP-1946, 1981.

3. Gl assman, A.J., "Computer Program for Design Analys is of Radial-Inflow Turbines, " NASA TN $0-8164,1976$.

4. Glassman, A.J., "Computer Program for Prel iminary Design Analys is of Axial-Flow Turbines," NASA TN D-6702, 1972.

5. Dolan, F.X., and Runstadler, P.W. Jr., "Pressure Recovery Performance of Conical Diffusers at High Subsonic Mach Numbers," NASA CR-2299, 1973.

6. Runstadler, P.W., Jr., "Pressure Recovery Performance of Straight-Channel, Single-Plane Divergence Diffusers at High Mach Numbers," Rept. N-88, Crear Inc., Hanover, $\mathrm{NH}$, (USAAVLABS-TR-69-56, AD-865300), Oct. 1969.
7. MCNally, W.D., "FORTRAN Program for Calculating Compressible Laminar and Turbulent Boundary Layers in Arbitrary Pressure Gradients, " NASA TN D-5681, 1970.

8. Bardina, J., Lyrio, A., Kline, S.J., Ferziger, J.H., and Johnston, J.P., "A Prediction Method for Planar Diffuser Flows," ASME Journal of Fluids Engineering, Vol. 103, 1981, pp. 315-321.

9. Ghose, S., and Kline, S.J., "The Computation of Optimum Pressure Recovery in Two-Dimensional Diffusers," ASME Journal of Fluids Engineering, Vol. 100, pp. 419-426.

10. Woolley, R.L., and Kline, S.J., "A Procedure for Computation of Fully Stalled Flows in TwoDimensional Passages," ASME Journal of Fluids Engineering, Vol. 100, pp. 180-186.

11. Ashjaee, J., and Johnston, J.P., "Straight-Walled, Two-Dimensional Diffusers-Transitory Stall and Peak Pressure Recovery, "ASME Journal of Fluids Engineering, Vol. 102, pp. 275-282.

12. Schumann, L.F., "A Three-Dimensional Axisymmetric Calculations Procedure for Turbulent Flows in a Radial Vaneless Diffuser," Journal of Engineering for Gas Turbines and Power, Vol. 108, No. 1, Jan. 1986, pp. 118-124.

13. White, F.M., Visous Fluid Flow, McGraw Hill, New York, 1974.

14. Shyne, R.J., Moore, R.D., and Boldman, D.R., "Comparison of Analytical and Experiments Performance of a Wind-Tunnel Diffuser Section," NASA TM-88795, 1986. 


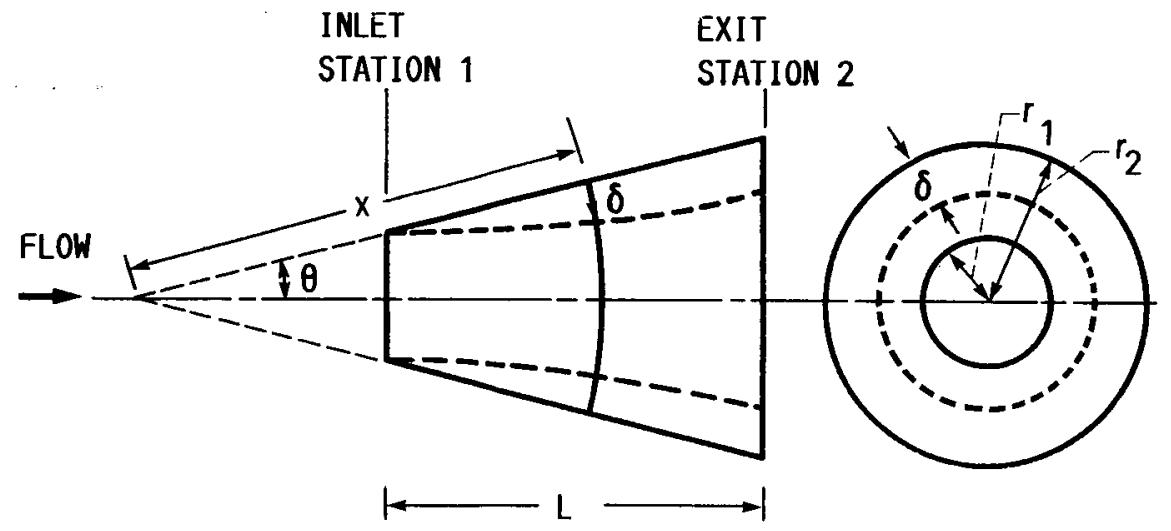

FIGURE 1.- CONICAL DIFFUSER GEOMETRY. 


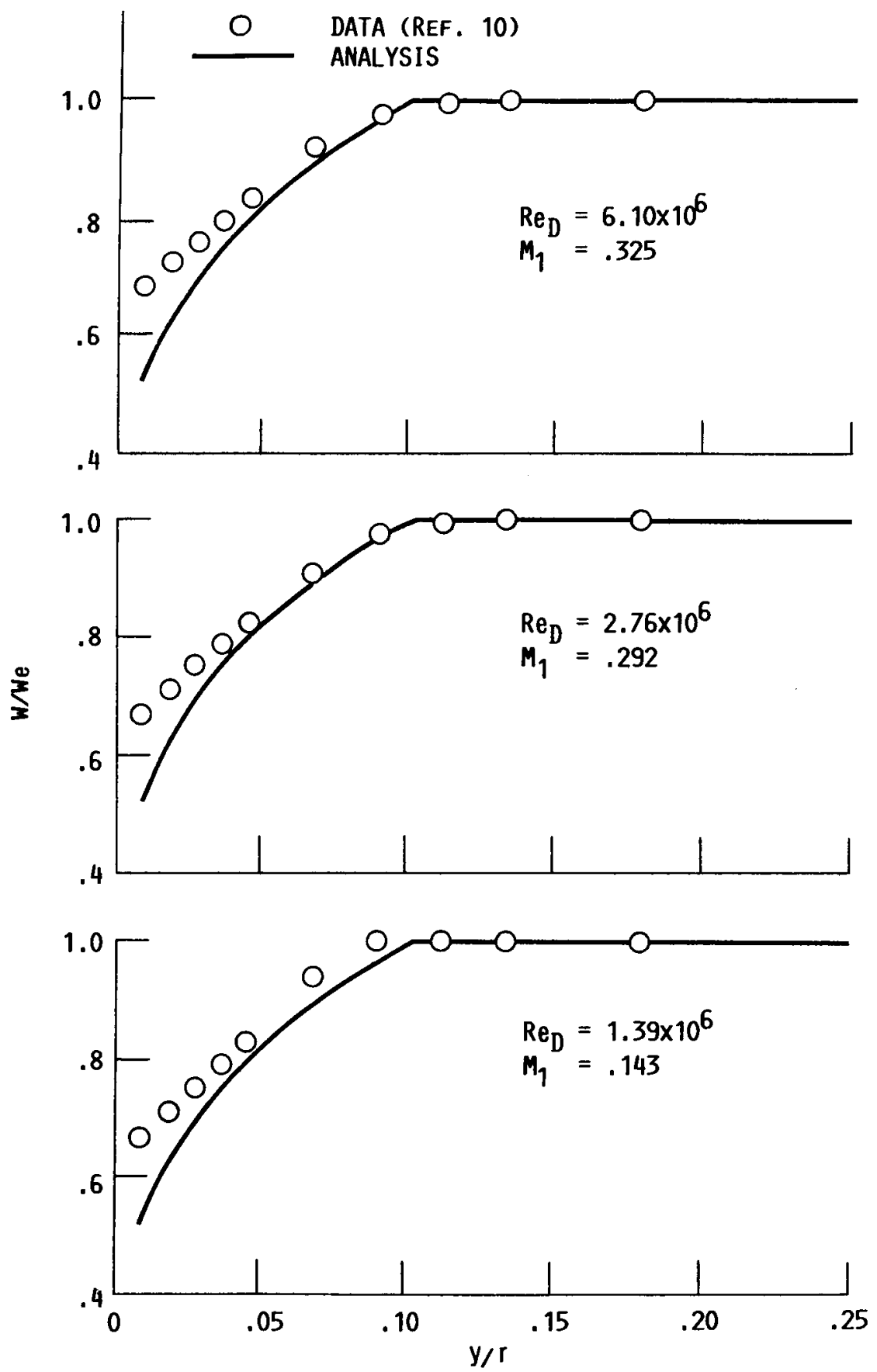

FIGURE 2.- BOUNDARY LAYER VELOCITY PROFILES AT EXIT OF .1 SCALE CROSSLEG DIFFUSER. 

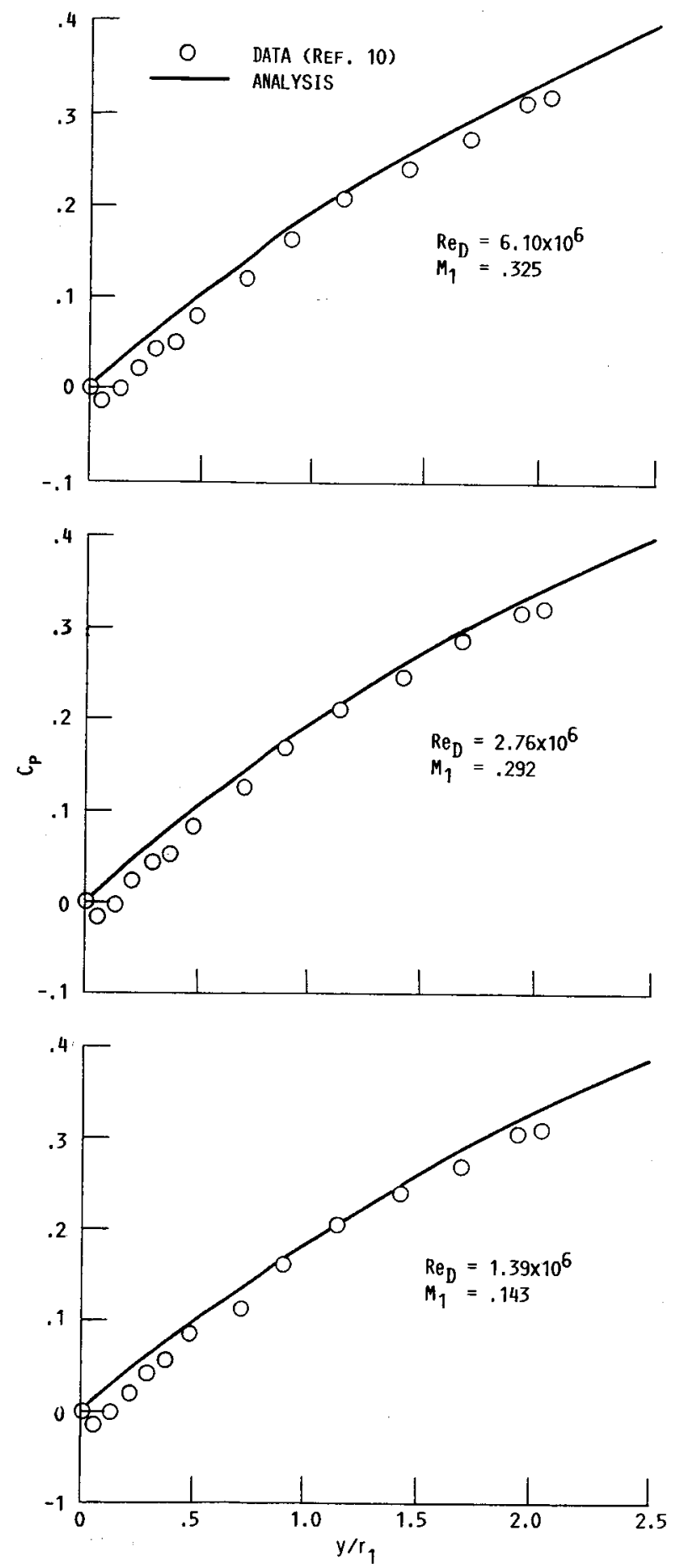

FIGURE 3.- PRESSURE RECOVERY COEFFICIENTS IN . 1 SCALE CROSSLEG DIFFUSER. 

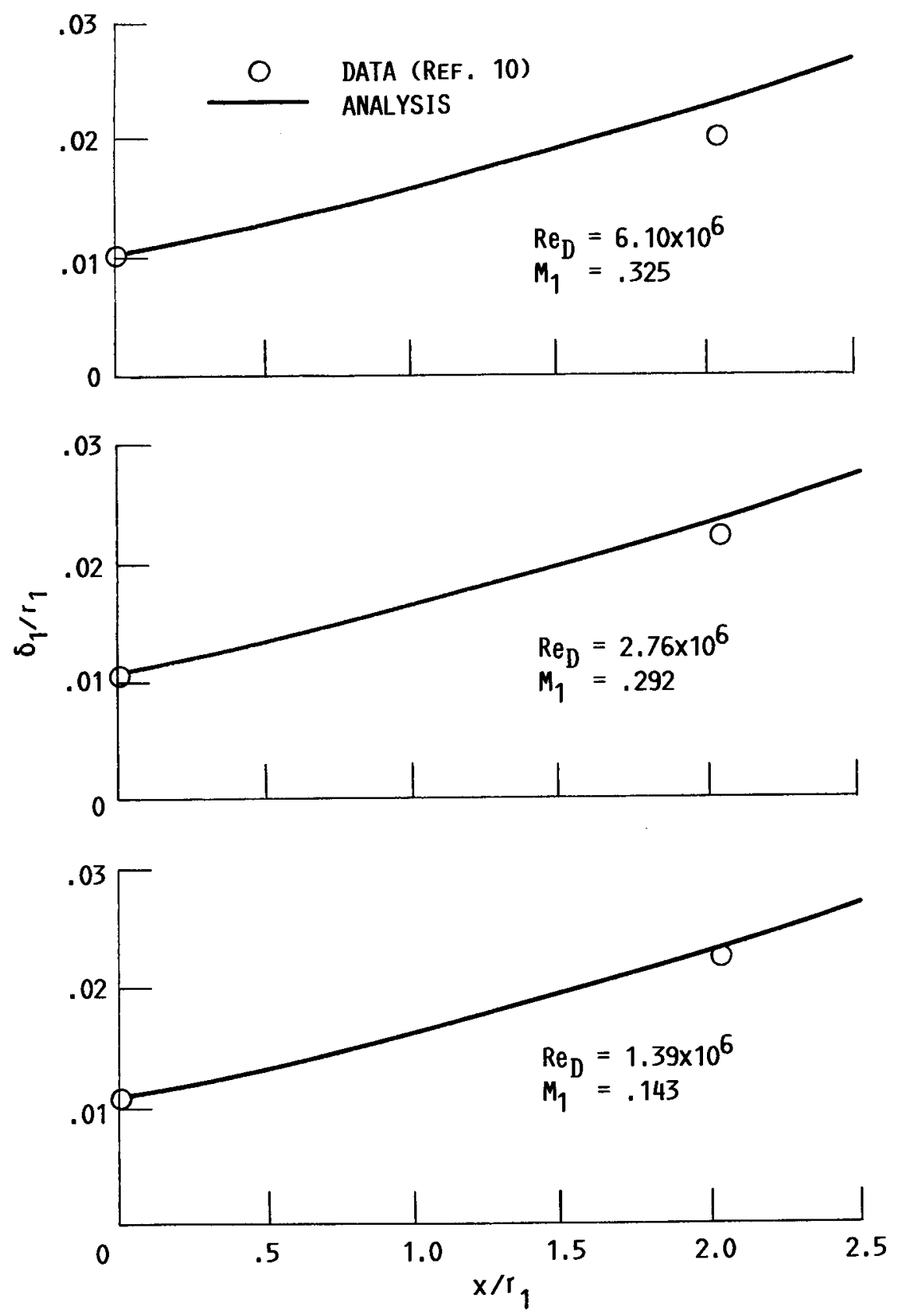

FIGURE 4.- DISPLACEMENT THICKNESSES IN . 1 SCALE CROSSLEG DIFFUSER. 


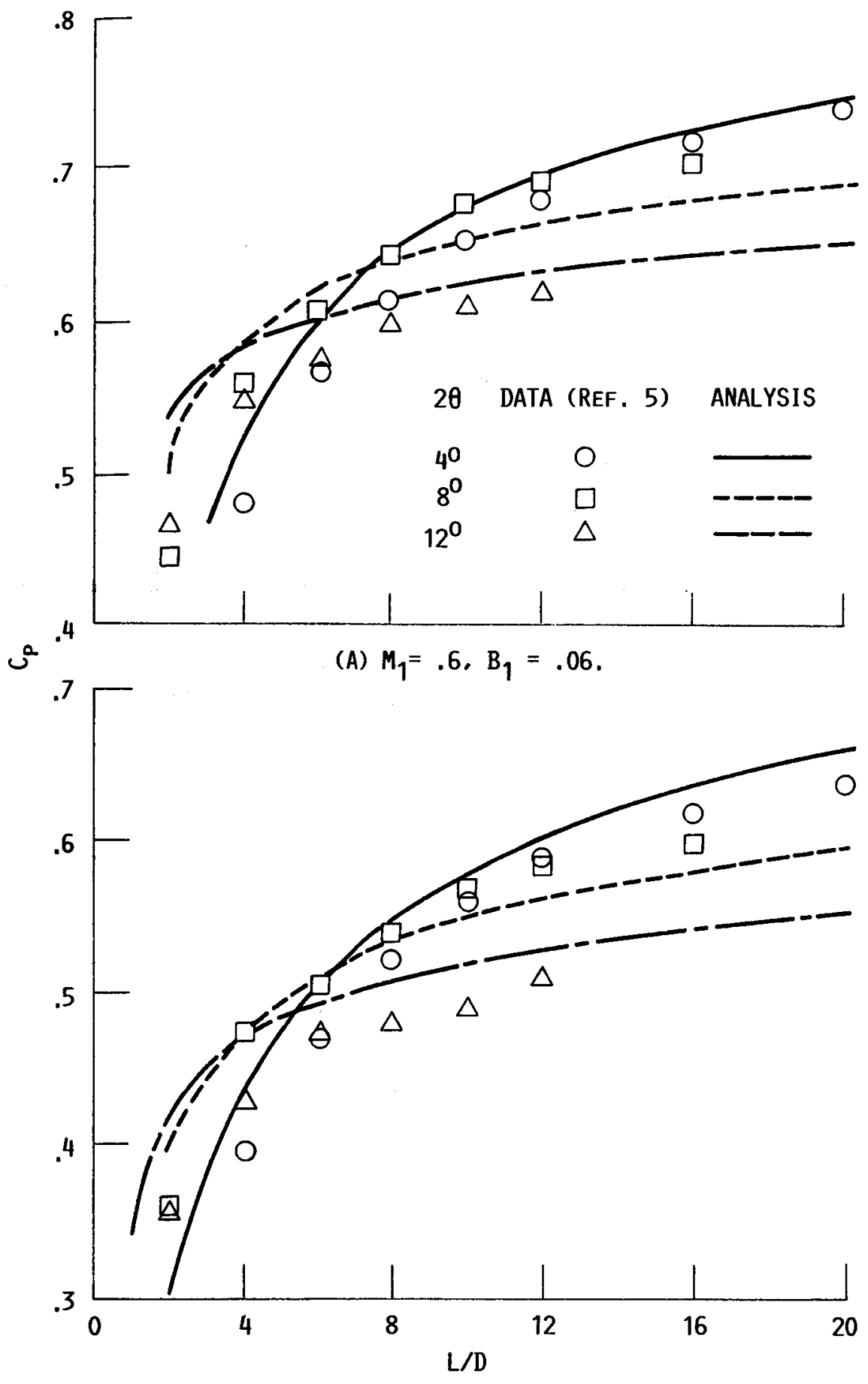

(B) $M_{1}=.6, B_{1}=.12$.

FIGURE 5.- PRESSURE RECOVERY COEFFICIENTS FOR VARIOUS CONICAL DIFFUSERS. 


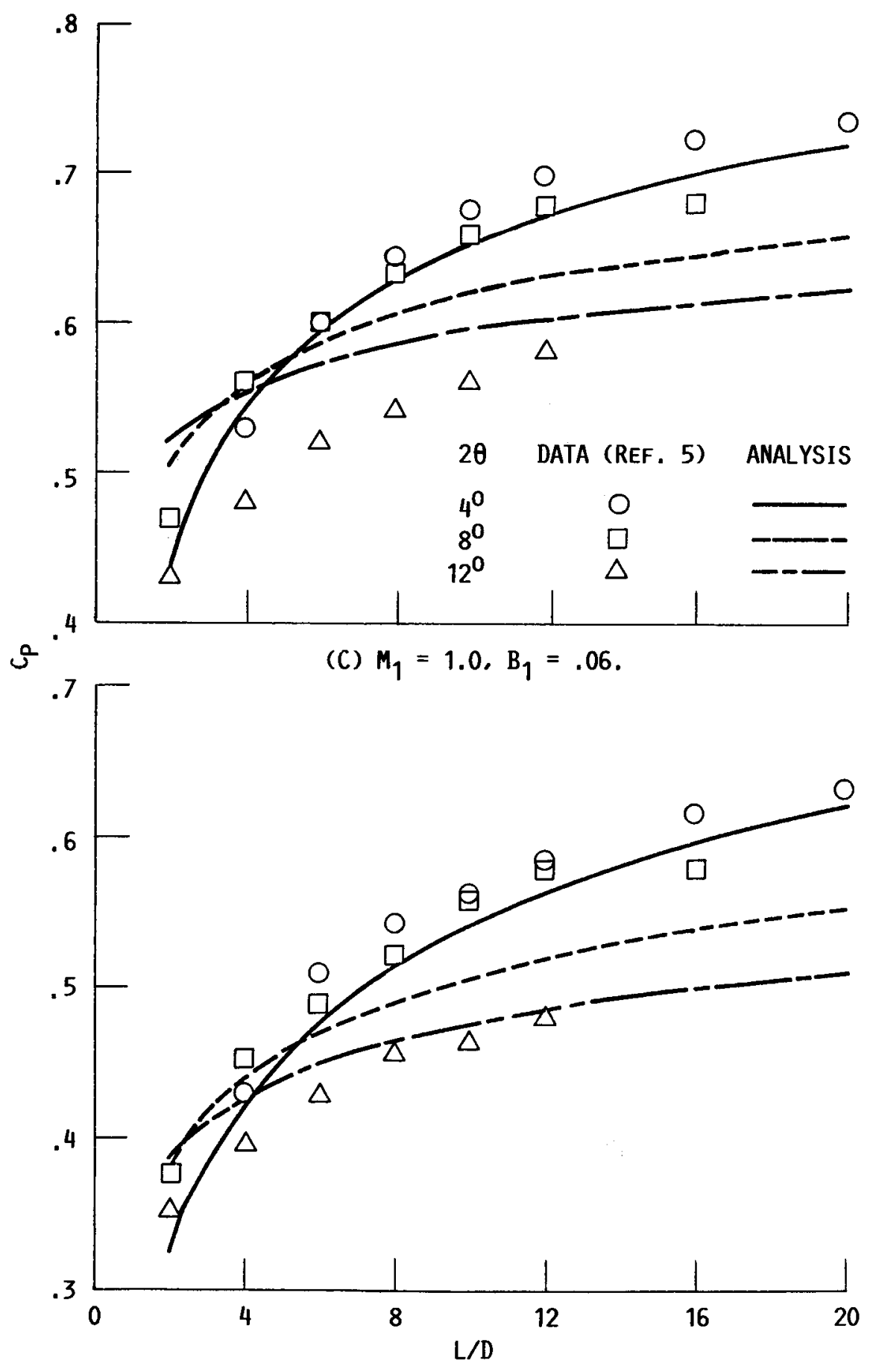

(D) $M_{1}=1.0, B_{1}=.12$.

FIGURE 5.- CONCLUDED. 


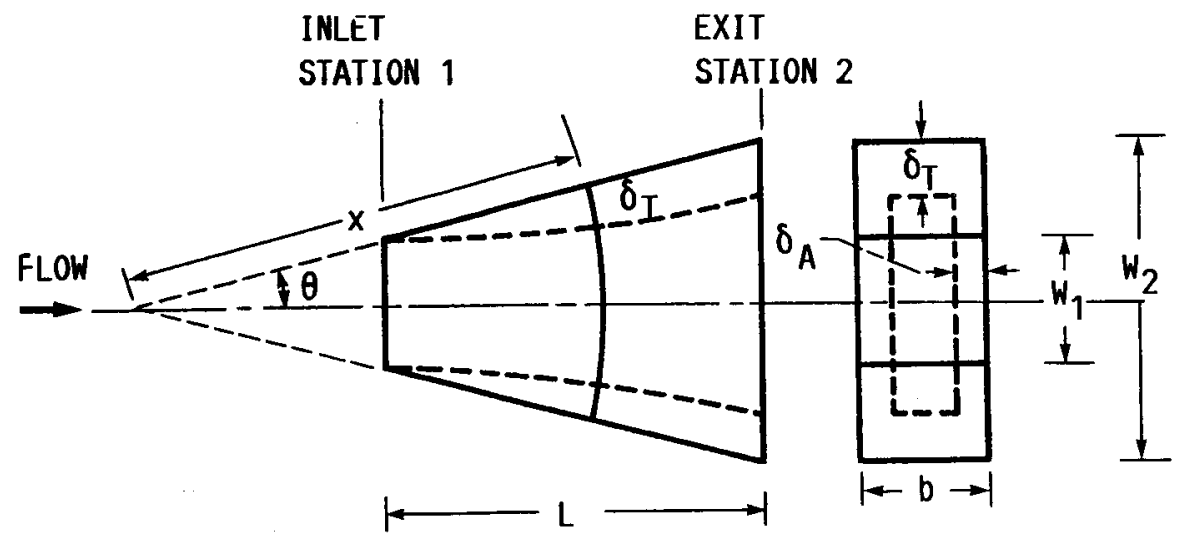

FIGURE 6.- RECTANGULAR DIFFUSER GEOMETRY. 

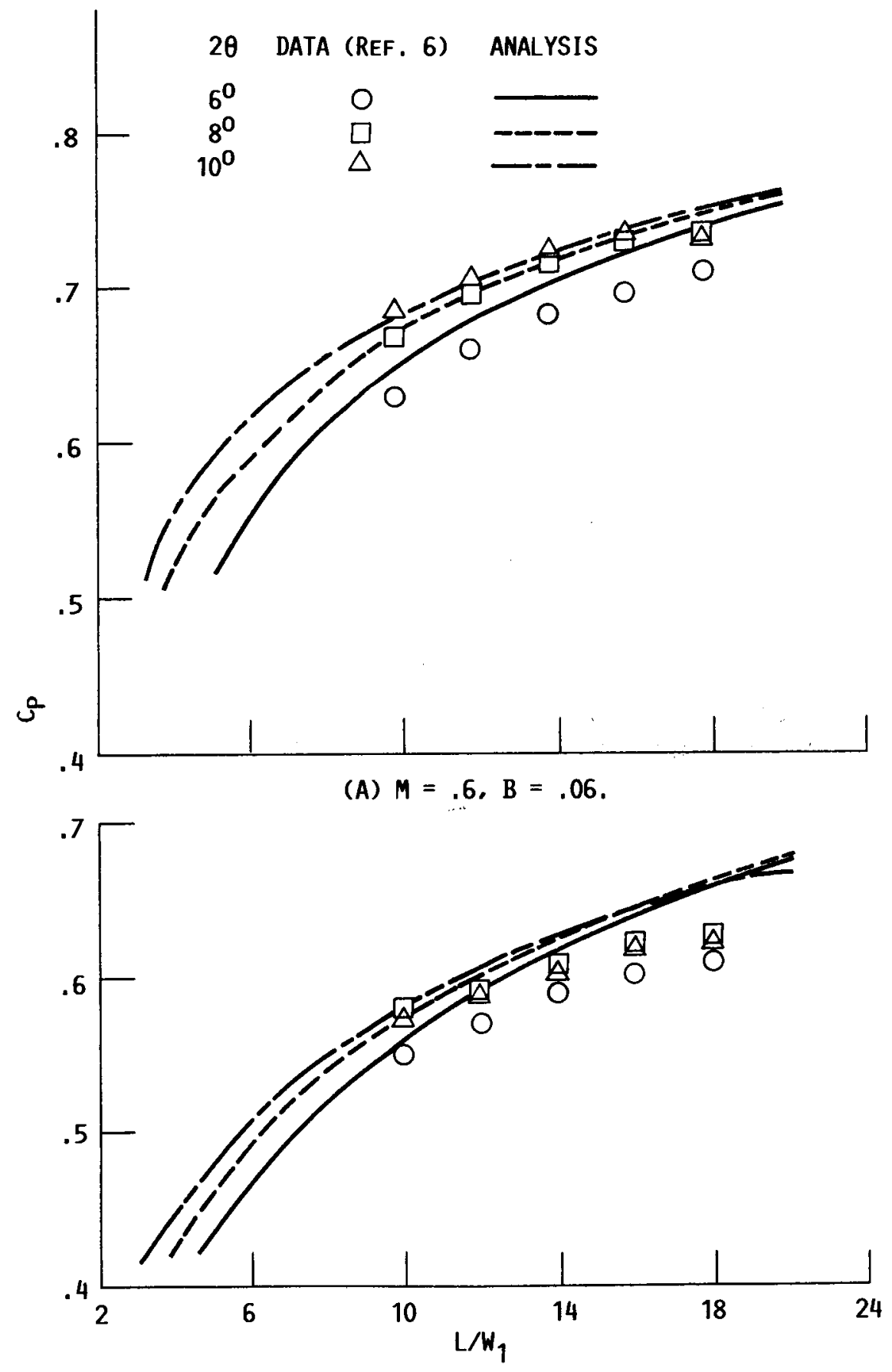

(B) $M_{1}=.6, B_{1}=.12$.

FIGURE 7. - PRESSURE RECOVERY COEFFICIENTS FOR VARIOUS RECTANGULAR DIFFUSERS. 

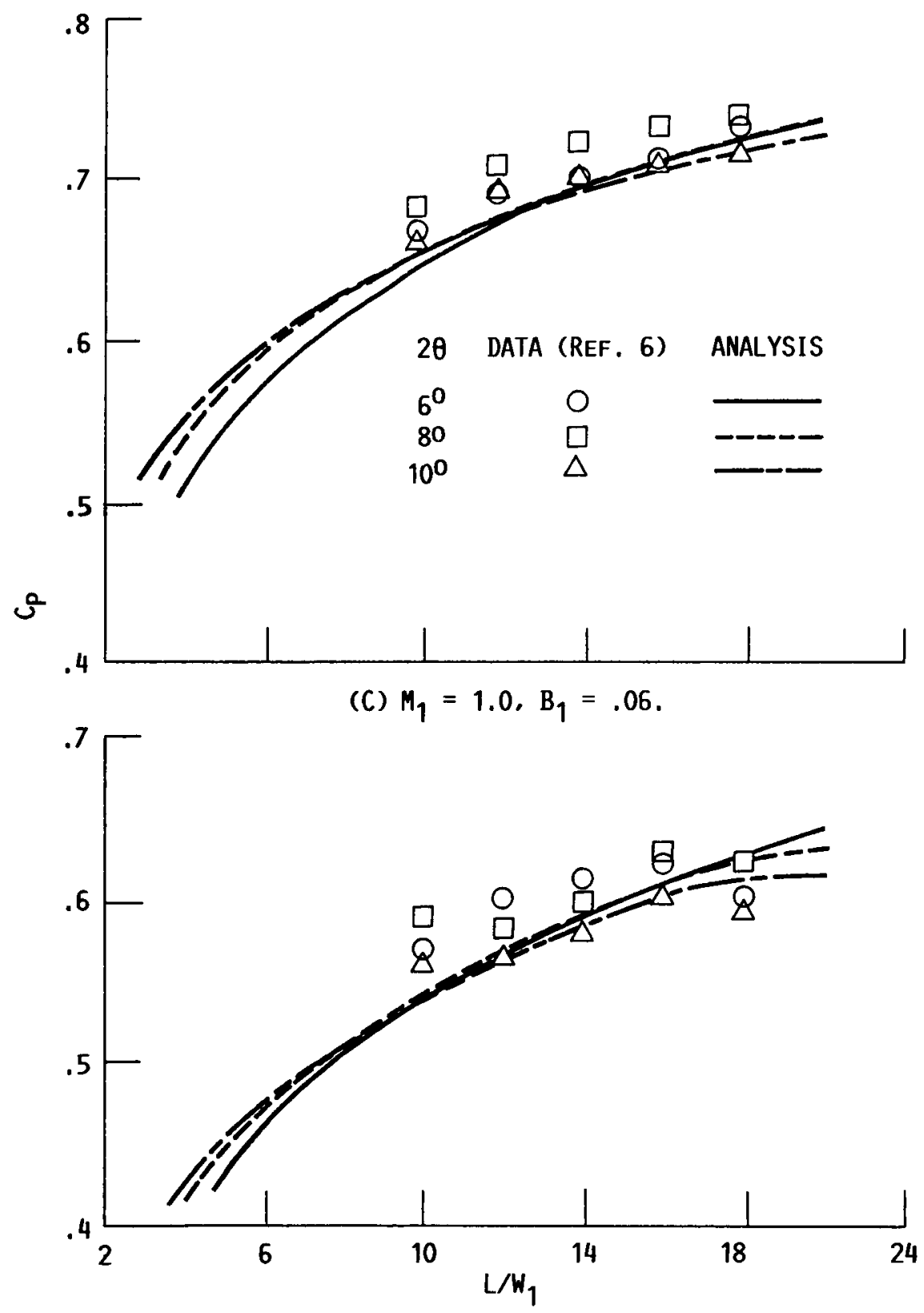

(D) $M_{1}=1.0, B_{1}=.12$.

FIGURE 7.- CONCLUDED. 


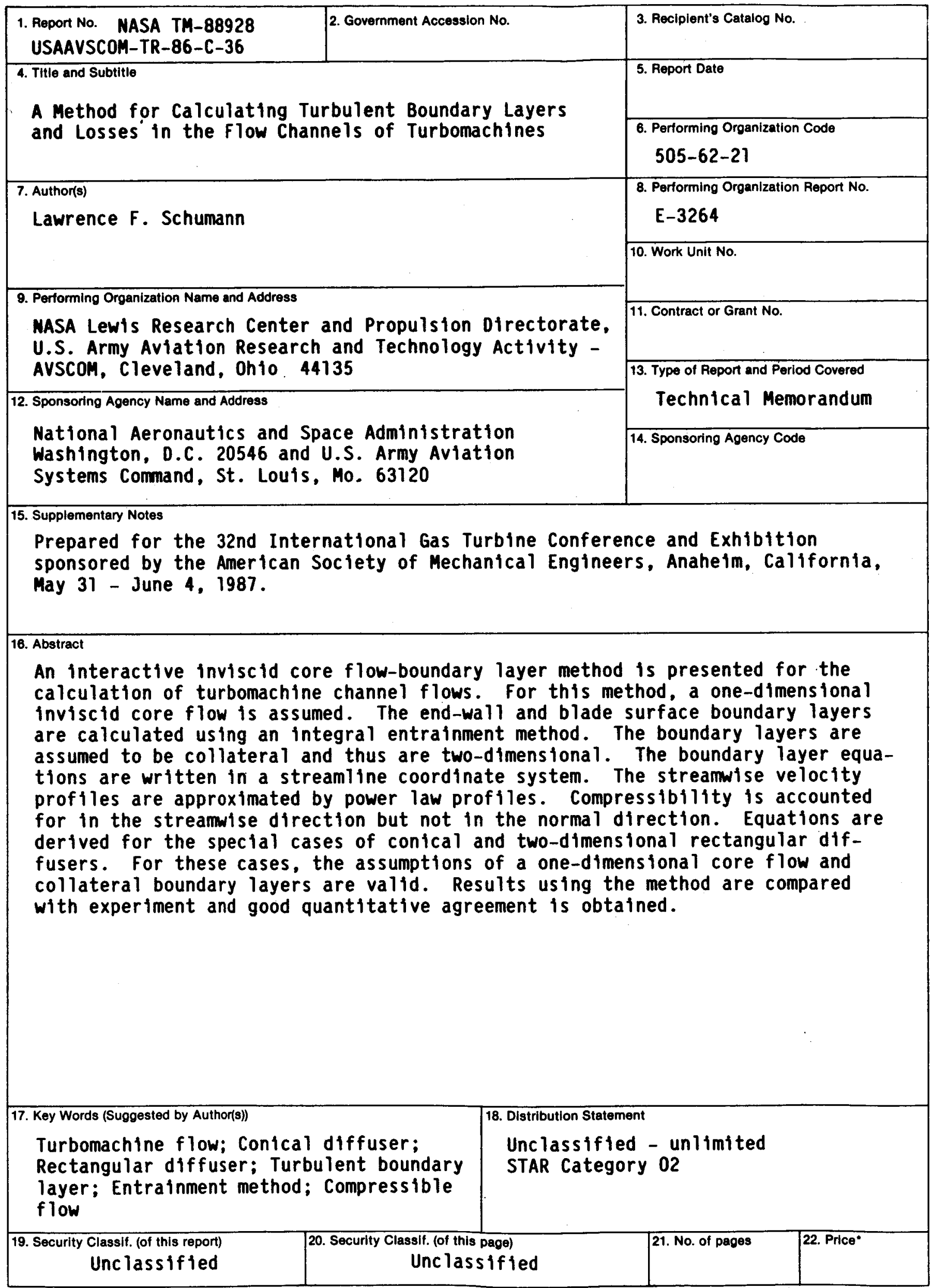

